\title{
Comparisons of Crosswind Velocity Profile Estimates Used in Fast-Time Wake Vortex Prediction Models
}

\author{
Mathew J. Pruis ${ }^{*}$, Donald P. Delisi ${ }^{\dagger}$ \\ NorthWest Research Associates, Redmond, Washington 98052 \\ Nash'at N. Ahmad \\ NASA Langley Research Center, Hampton, Virginia 23681
}

\begin{abstract}
Five methods for estimating crosswind profiles used in fast-time wake vortex prediction models are compared in this study. Previous investigations have shown that temporal and spatial variations in the crosswind vertical profile have a large impact on the transport and time evolution of the trailing vortex pair. The most important crosswind parameters are the magnitude of the crosswind and the gradient in the crosswind shear. It is known that pulsed and continuous wave lidar measurements can provide good estimates of the wind profile in the vicinity of airports. In this study comparisons are made between estimates of the crosswind profiles from a priori information on the trajectory of the vortex pair as well as crosswind profiles derived from different sensors and a regional numerical weather prediction model.
\end{abstract}

\section{Introduction}

$\mathrm{F}$ ive methods for estimating the vertical crosswind profile near the terminal area are compared in this study. Information on current winds and an accurate short-term forecast is crucial for driving wake vortex decay and transport models ${ }^{1,2,3}$, and important for running the terminal area operations in general. Ultimately good wind estimates and an understanding of the uncertainties associated with these wind estimates will enable improved operations ${ }^{4}$ in the terminal area. For example, the ability to operate simultaneously on closely spaced parallel runways - through increased accuracy in surveillance and navigation, and through improved understanding of wake vortices and weather conditions - means airports will gain capacity, enhance safety and improve environmental performance $e^{4,5}$.

The focus of this study is on crosswind estimates used to drive fast-time wake vortex prediction models. The small spatial and temporal scales of wake physics necessitate very high-resolution spatiotemporal estimates of the winds at the height of wake vortices. Ideally the wind predictions would be on the order of tens of meters over a region which may cover several kilometers and be available every few minutes ${ }^{6}$. These spatial and temporal scales are a challenge to obtain with current technologies.

Methodologies for short-term weather estimation can be broadly divided into two categories: nowcasting and mesoscale modeling. Nowcasting is based on time-averaged in-situ observations from wind sensors such as lidar wind scanners or anemometers. Data from different measurements can be assimilated by nowcasting tools and extrapolated to provide a short-term forecast ${ }^{7}$. Some nowcasting tools combine the observations with simulation data from mesoscale models. The disadvantage in the nowcasting methodology is the high cost of deploying the large number of sensors required to adequately cover the spatial extant of the terminal area and the uncertainty introduced by the need to interpolate or extrapolate away from the sensor locations.

Short-term weather estimates can also be obtained by using high-resolution regional atmospheric models. Substantial strides have been made in the mesoscale modeling research over the last three decades and a number of mesoscale models are in current operational use. These models include the National Center for Atmospheric Research's (NCAR) state-of-the-art Weather Research and Forecasting (WRF) model ${ }^{8}$, Science Applications International Corporation's Operational Multiscale Environment model with Grid Adaptivity ${ }^{9}$ and the United States Navy's Coupled Ocean/Atmosphere Mesoscale Prediction System ${ }^{10}$. The mesoscale models may include data

\footnotetext{
${ }^{*}$ Research Scientist, NWRA, $4118148^{\text {th }}$ Ave NE, Redmond, WA 98052. Member, AIAA.

${ }^{\dagger}$ Senior Research Scientist, NWRA, $4118148^{\text {th }}$ Ave NE, Redmond, WA 98052. Senior Member, AIAA.

" Research Aerospace Engineer, NASA, Hampton, Virginia. Senior Member, AIAA.
} 
assimilation or a frequent re-initialization of the model such as National Centers for Environmental Prediction's (NCEP) Rapid Update Cycle (RUC) model $^{11}$. Some recent applications of mesoscale modeling to support terminal area operations and wake vortex transport prediction include Refs 12-15. There are two major disadvantages in using mesoscale models for wake vortex applications. First, the computational cost of running the models at the appropriate spatial and temporal resolution for wake applications can be prohibitive. Second, the physics parameterizations in these models have been designed for mesoscales (horizontal grid resolution on the order of tens of kilometers) and not for horizontal resolutions of tens of meters. Operational mesoscale models running at their highest grid resolution $(1 \mathrm{~km}-10 \mathrm{~km}$ in the horizontal) can at best resolve the whole terminal area by just a few grid points. This grid resolution is coarse for wake applications and can introduce uncertainties in the wind estimates. The grid resolution related uncertainties are in addition to the uncertainties inherent in the model due to the nonlinearity of the underlying mathematics as well as incompleteness of initial and boundary conditions.

In this study several methods of estimating the vertical crosswind profile are compared with estimates of timeaveraged crosswind profiles obtained using a Lockheed Martin Coherent Technologies (LMCT) pulsed lidar. Two of the methods use a priori information on the trajectory of the vortex pair. These are compared with the results from assuming the 10-m height winds measured using the airport's Automated Surface Observing System (ASOS) wind sensor are constant with height and also the predictions of a regional numerical weather prediction model.

This study has yielded several important observations. First, with a lidar wind scanner available, different methods can provide estimates of the average crosswinds at the vortex height with mean biases that are less than 30 $\mathrm{cm} / \mathrm{s}$. The two methods which do not utilize a lidar wind scanner, that is linearly extrapolating ASOS wind measurements to height and simulations of a regional numerical weather prediction model, have substantially larger standard deviations in their bias estimates (up to $2.5 \mathrm{~m} / \mathrm{s}$ ) than other methods which utilize lidar observations (standard deviations of less than $55 \mathrm{~cm} / \mathrm{s}$ ). Large variances in the crosswind bias indicate substantial uncertainty on the mean wind estimate from landing to landing, even if the mean bias of the entire dataset is small. Second, a measure of the temporal variability after correcting for the bias or a mean shift of the wind profile, at the heights of the wake vortices (up to $325 \mathrm{~m}$ in this study) and over the time intervals that wake vortices are observed (typically $50-180$ seconds), has a mean of less than $20 \mathrm{~cm} / \mathrm{s}$. This temporal variability is approximately the same magnitude as the spatial variability in the vertical crosswind profile over the heights traversed by the vortices during the observation time interval. Thus both the mean shift of the wind and changes in the shape of the wind profile are important when estimating uncertainties in crosswind estimates relevant to wake vortex studies.

There are additional methods of nowcasting or estimating current wind conditions near airports which were not included in this study; for example, extrapolating ASOS wind estimates to height using a simple boundary layer model, other mesoscale models, or other wind sensors. This study is not intended to be comprehensive of all potential crosswind estimation methods; instead it is intended to provide insight into expected variability of crosswinds relevant to wake vortex physics and wake turbulence mitigation, and to provide a baseline from which other crosswind estimation methods can be compared.

\section{Data set description}

Field experiments to examine wake vortex behavior are frequently performed with either a pulsed or continuous wave lidar on-site. These field experiments therefore have available vertical profiles of the wind available at the relevant temporal and spatial scales for wake vortex studies. Four lidar data sets are included in this study. These data sets were obtained with the purpose of studying wake vortex behavior. All of the lidar data was collected with a Lockheed Martin Coherent Technologies (LMCT) pulsed lidar. Two of the data sets were obtained at the Denver International Airport (DEN), one was collected at the San Francisco International Airport (SFO), and the final data set was collected at Lambert-St. Louis International Airport (STL).

The data set collected at the highest initial vortex height was at DEN and was collected from April 5 through June 6, 2006. The average height of the generator aircraft is approximately 275 meters. There are 4372 landings included in the study. The average observation lifetime is 41 seconds with a standard deviation of 28 seconds. The maximum observation lifetime for the data set is 190 seconds.

The data set with the second highest initial vortex generation height is also at DEN, but collected three years earlier from August 25 through September 20, 2003. The mean initial vortex height for this data set is approximately 220 meters. There are 1966 landings included in the data set. The average observation lifetime is 63 seconds with a standard deviation of 30 seconds. The maximum observation lifetime is 233 seconds.

The third data set was also collected in the out of ground effect region at an initial vortex height of 152 meters. This data set was collected at SFO from September $9-24,2001$. There are 2151 landings in this data set. The 9/11 terrorist attacks occurred during this time interval which led to a temporary closure of the US airspace. 
Subsequently, no landings were observed with the lidar at SFO from 07:16 PDT on September 11th to 17:27 PDT on September 13th. The mean observation lifetime for this data set was 65 seconds with a standard deviation of 28 seconds. The maximum observed lifetime is 241 seconds for this data set.

The final data set was collected at STL with the wake observations spanning a longer time interval than the other studies. Observations in this data set were obtained from July 1, 2003, to January 31, 2005. Although the observation interval is longer, a similar number of landings (2237) are used. The mean initial height of this data set is 60 meters. The mean observation lifetime for the data set is 64 seconds with a standard deviation of 27 seconds. The maximum observation lifetime is 289 seconds.

\section{Crosswind estimation methods}

In this paper five methods of estimating the crosswind are compared for four different wake vortex field experiments. Comparing the crosswind estimation methods in detail provides insight into the estimation error of each method, and also insight into the uncertainties in the meteorological sensing of the crosswinds due to nonhomogeneity of weather conditions and changes in the weather since time of last observation.

\section{A. Time-averaged lidar crosswinds}

The four data sets used in this study all have crosswind profile estimates taken before and after the vortex observation period. Typically when these observations are used for driving fast-time wake vortex numerical models, the two wind profiles are averaged to obtain a single vertical crosswind profile. This timeaverage lidar estimate of the crosswind is used as the baseline crosswind profile for this study. All other crosswind estimation methods are compared directly against the lidar time-averaged crosswinds.

The LMCT lidar actually estimates winds in the plane of the lidar scan. However, since a lidar is typically oriented such that the scan plane is perpendicular to the aircraft approach path, the lidar inplane winds are estimates of the crosswind. Lidar inplane winds are obtained using the radial velocities measured along successive scans which are then interpolated to a vertical height grid and averaged (Figure 1). The vertical crosswind profiles therefore represent an average of the crosswinds over the width of

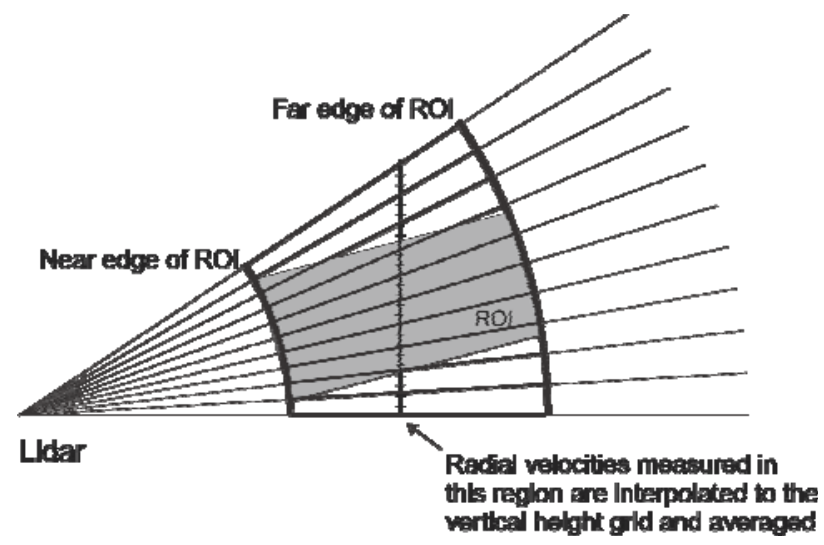

Figure 1. Lidar in-plane winds are obtained using the radial velocities measured along successive scans. The radial velocities are interpolated to a vertical height grid and averaged over the Region of Interest (ROI) to obtain a single vertical profile.

a pre-determined region of interest (ROI) and a vertical dimension related to the separation of the arcs over the time it takes to complete a scan, typically less than 5 seconds. Since the baseline method averages the before and after winds, an additional averaging period is introduced which is variable depending on the observation interval. The observation interval is typically 40 to 70 seconds, but can be several minutes.

\section{B. Bilinear interpolated crosswinds}

To overcome the shortcomings of the ambiguity introduced by changes in the wind conditions over the observation time interval, a new method for estimating winds using a priori information from the lidar vortex trajectories is suggested. The LMCT aircraft wake vortex algorithm was used to estimate the horizontal and vertical locations of the vortex pair over time for the data sets used in this study. The a priori information on the vortex vertical position can be used for bilinear interpolation of the lidar crosswind profiles obtained before and after the vortex observation interval to better estimate the winds encountered by the vortex pair. The method is based upon the assumption that the winds at the start of the observation interval should be more similar to the wind profile measured before the observation interval, while the winds experienced by the wake vortices near the end of the observation interval should be more similar the winds measured after the observation interval. Figure 2 shows one example comparing the bilinear interpolated wind estimate with the time-averaged lidar crosswind estimate. 

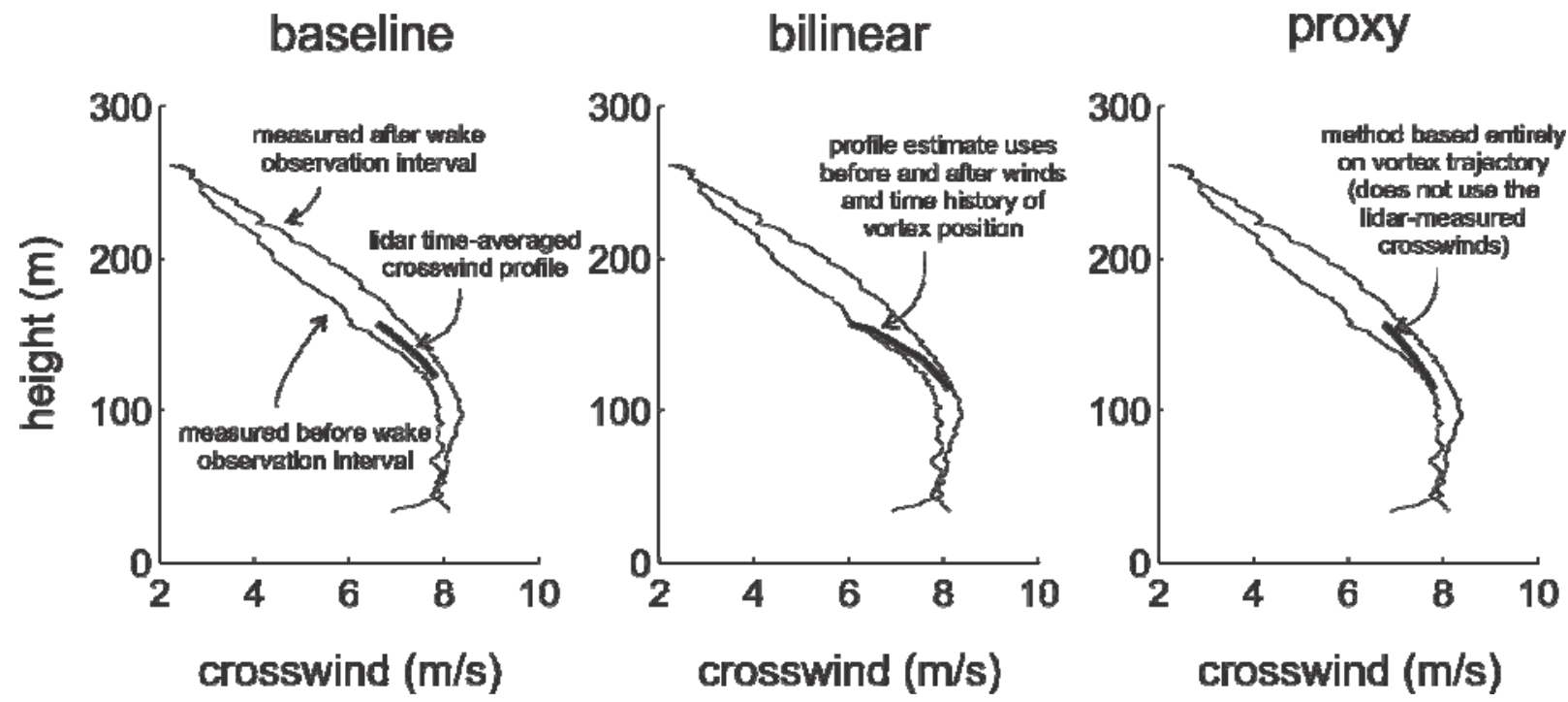

Figure 2. Example of the crosswind estimates from the methods based upon in-situ lidar observations for a single landing at SFO. The thin black lines are the vertical profiles of crosswind measured by the lidar before and after the wake vortex observation period. For this landing, there are $\mathbf{3 0 . 3}$ seconds between the two wind observations. The thick black line is the estimate of the crosswind profile at the heights where a wake vortex was tracked by the lidar. The crosswind profiles can be compared against one another by calculating the mean shift or bias and the variance or standard deviation over the heights where the vortices were observed. The bilinear interpolation method has a bias of $0.13 \mathrm{~m} / \mathrm{s}$ with respect to the baseline and a standard deviation of $0.28 \mathrm{~m} / \mathrm{s}$. For this example, the proxy method predicts a vertical velocity profile that more closely matches the baseline in both the mean and also in the shape or form of the profile. The bias, or mean shift, of the proxy crosswind profile compared to the baseline is $-0.03 \mathrm{~m} / \mathrm{s}$ and the standard deviation is $0.13 \mathrm{~m} / \mathrm{s}$.

\section{Proxy crosswinds}

It is also possible to use the lidar trajectories to directly estimate the crosswinds encountered by the vortex pair if some assumptions are made about vortex physics. In the out of ground effect region, fast-time wake vortex models transport the vortices laterally with the crosswind. Some models slightly modify this transport in the presence of a crosswind shear gradient, but, to first order, estimates of the crosswind can be obtained directly from the wake vortex trajectories as $\partial \mathrm{Y}(\mathrm{z}) / \partial \mathrm{t}+$ error, where $\mathrm{Y}$ is the lateral position of the vortices and $\mathrm{t}$ is time. Both the port and starboard vortices can be treated as independent tracers to estimate the vertical crosswind profile. To convert these independent (and frequently noisy) point estimates of the crosswind into a single smoothed vertical profile, we used a smoothing spline ${ }^{16}$ with the desired equivalent number of degrees of freedom (trace of the smoother matrix) set equal to five. An example of this method compared with the before and after lidar wind estimates is shown in Figure 2 .

We have also applied the proxy crosswind method to wake vortices measured in the in ground and near ground effect regions (for example, at STL). Interaction of the wake vortices with the surface modifies the lateral transport of the vortex pair in this region and the vortices in the fast-time models no longer move laterally solely as a function of the crosswinds. Nonetheless we have found that the proxy crosswind still produces reliable estimates of the crosswind over the period when both the port and starboard vortices are observed. This is because the modifications to the lateral motion of the vortex pair due to the surface is, to first order, approximately equal but in the opposite direction for two vortices. Ground effects tend to increase the scatter in the individual estimates, but only have a small effect on the mean crosswind profile.

\section{ASOS crosswinds}

The Automated Surface Observing Systems (ASOS) program is a joint effort of the National Weather Service (NWS), the Federal Aviation Administration (FAA), and the Department of Defense (DOD). ASOS provides information on the wind direction, speed and character (e.g., gustiness). ASOS reports a 2-minute average of 5second average wind directions and speeds every minute. Typical ASOS wind sensor heights are 33 feet or 27 feet, depending on local site-specific restrictions or requirements. For this study, we have used the ASOS wind estimates 
Table 1. Comparison of the mean shift or bias, and its standard deviation, in the crosswind for each of the methods as compared to the baseline crosswind.

\begin{tabular}{|c|c|c|c|c|c|c|c|c|}
\hline & \multicolumn{2}{|c|}{ bilinear } & \multicolumn{2}{|c|}{ proxy } & \multicolumn{2}{|c|}{ ASOS } & \multicolumn{2}{|c|}{ MM5 } \\
\hline & $\begin{array}{c}\text { Mean } \\
\text { bias } \\
(\mathrm{m} / \mathrm{s})\end{array}$ & $\begin{array}{c}+/- \\
\text { std } \\
(\mathrm{m} / \mathrm{s})\end{array}$ & $\begin{array}{c}\text { Mean } \\
\text { bias } \\
(\mathrm{m} / \mathrm{s})\end{array}$ & $\begin{array}{c}+/- \\
\text { std } \\
(\mathrm{m} / \mathrm{s})\end{array}$ & $\begin{array}{c}\text { Mean } \\
\text { bias } \\
(\mathrm{m} / \mathrm{s})\end{array}$ & $\begin{array}{c}+/- \\
\mathrm{std} \\
(\mathrm{m} / \mathrm{s})\end{array}$ & $\begin{array}{c}\text { Mean } \\
\text { bias } \\
(\mathrm{m} / \mathrm{s})\end{array}$ & $\begin{array}{c}+/- \\
\mathrm{std} \\
(\mathrm{m} / \mathrm{s})\end{array}$ \\
\hline DEN'06 $(n=4372)$ & 0.00 & 0.06 & -0.11 & 0.43 & -0.66 & 2.40 & & \\
\hline DEN'03 $(n=1966)$ & 0.00 & 0.06 & 0.05 & 0.45 & & & & \\
\hline $\operatorname{SFO}(n=2151)$ & 0.00 & 0.06 & -0.12 & 0.33 & & & 3.87 & 2.48 \\
\hline STL $_{(n=2237)}$ & 0.00 & 0.07 & 0.29 & 0.51 & -0.08 & 0.92 & & \\
\hline
\end{tabular}

at DEN during the 2006 experiment and also the STL experiment. The ASOS winds were interpolated to the start of the observation interval and it was assumed the ASOS wind was constant with height.

\section{E. MM5 numerical weather prediction simulation crosswinds}

For this study, a Mesoscale Model 5 (MM5) simulation was performed over the interval of the SFO experiment. The MM5 simulation setup consisted of three computational domains. The outermost domain was bounded between $18^{\circ} \mathrm{N}$ to $50^{\circ} \mathrm{N}$ in latitude and $145^{\circ} \mathrm{W}$ to $75^{\circ} \mathrm{W}$ in longitude. The outermost computational domain had a grid resolution of $36 \mathrm{~km}$. Two higher resolution nests were defined within the outermost domain. The innermost domain centered over San Francisco had a grid resolution of $4 \mathrm{~km}$. Five minute resolution terrain and landuse datasets were use for the two outermost domains and 2 minute resolution terrain and landuse datasets were used for the innermost nest. A total of 47 vertical levels were defined with the first level at 3m AGL. The simulations were initialized using the ETA-NAM model data from the National Climate Data Center (NCDC) at 00Z for the dates starting at September $9^{\text {th }}, 2001$ through September $29^{\text {th }}, 2001$ and run for a 24-hour forecast for each day. The boundary conditions data was ingested into the model run at $3 \mathrm{hrs}$ intervals. The MM5 model was developed by the Pennsylvania State University and the National Center for Atmospheric Research ${ }^{17}$. The MM5 simulation data set provided an estimate of the crosswind velocity profile every 30 minutes. These estimates were interpolated in time to the start of the observation interval (wake age $=0$ ).

\section{Results}

To compare crosswind profile estimation techniques we use the time-average of the before and after lidar winds as the baseline. The time-average lidar crosswind is commonly used by wake modeling groups when driving fasttime wake vortex models for comparison with lidar wake vortex observations. Each crosswind estimation technique is compared to the baseline using simple descriptive statistics to examine how similar or different the estimates are from each other, and to look for insights into the uncertainties in the meteorological sensing of the crosswinds due to nonhomogeneity of weather conditions and changes in the weather since time of last observation.

\section{A. Mean bias, or mean shift, in crosswind}

Table 1 shows the mean bias, or shift, in the crosswind profile for four of the methods compared with the baseline. As seems reasonable, the bilinear interpolation method of estimating the crosswinds, which is generated using the before and after lidar crosswind estimates as well as the time history of vortex vertical position, does not have a mean bias compared to baseline crosswinds at any of the study areas. The standard deviation of the distribution of the bias at each site is also comparatively small at 0.06 to $0.07 \mathrm{~m} / \mathrm{s}$.

In comparison, the mean bias and its standard deviation are substantially larger for the proxy crosswind estimation technique. This is interesting because the proxy method, which is estimated using lidar-observed vortex trajectories and does not include lidar-estimated crosswinds, may be the most relevant crosswind to use in wake vortex modeling studies.

The ASOS wind estimates have mean biases of -0.08 and $-0.66 \mathrm{~m} / \mathrm{s}$. The standard deviation in the ASOS bias estimates is much larger than either the bilinear interpolation or proxy methods at 0.92 and $2.4 \mathrm{~m} / \mathrm{s}$. As should be expected, the ASOS wind estimates at STL, where the vortex heights are less than 120 meters above the surface, are better than the ASOS wind estimates are for the DEN data set in 2006 where the vortex heights range up to 325 meters above the surface. 
Table 2. Comparison of the bias, or mean shift, of the proxy crosswind with respect to the baseline crosswind estimate, as a function of baseline crosswind speed. The whiskers on the box plots show the sample minimum and maximum, the box shows the range between the lower quartile and upper quartile and contains $50 \%$ of the data with $25 \%$ of the residuals above and $25 \%$ of the residuals below, and the red line indicates the sample mean. The darker gray line is the median. Frequently the red line (sample mean) overlies the darker gray line (sample median) in the box plots. The data has not been processed to remove outliers in the wake vortex observations or in the crosswind estimates.

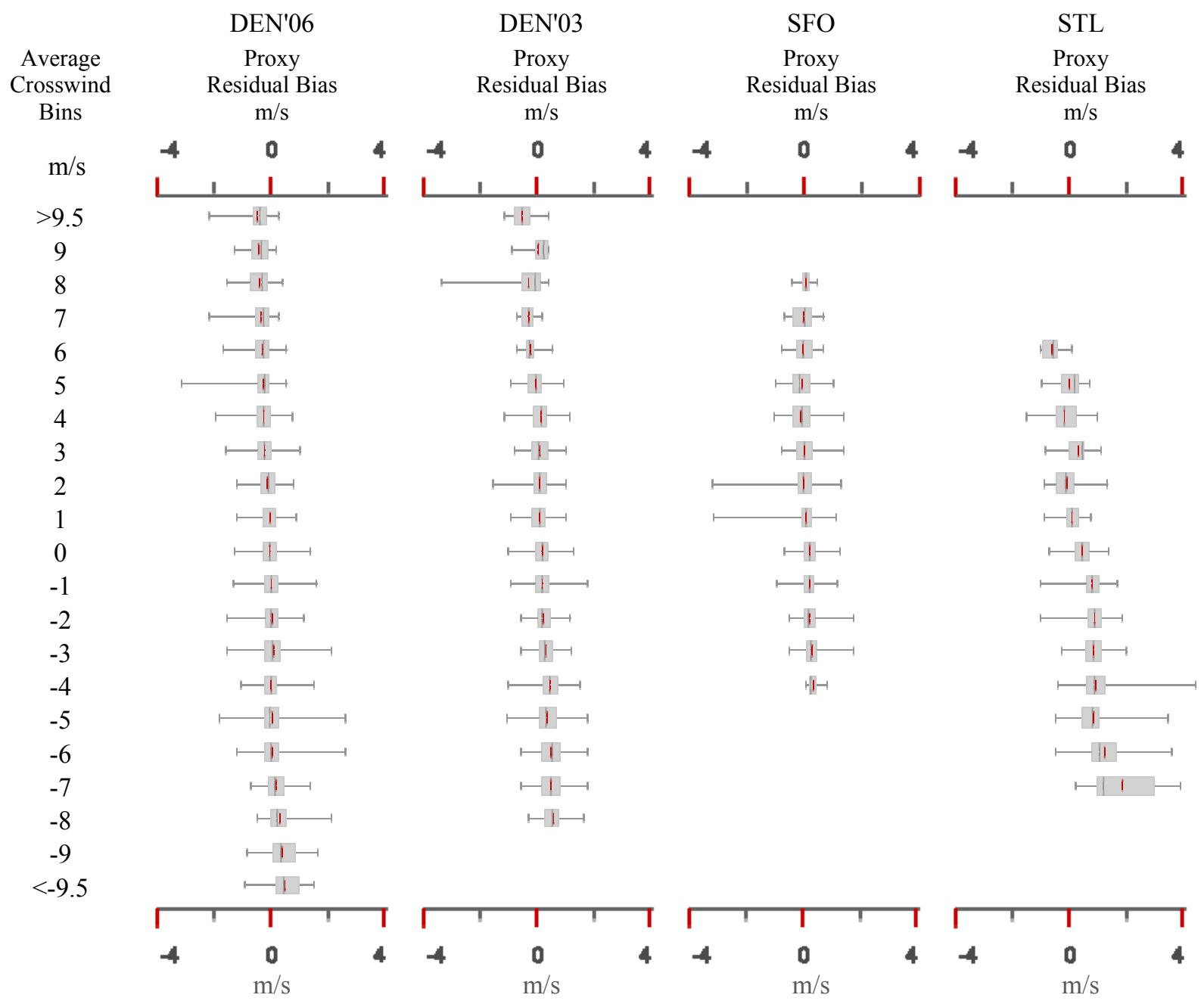

Lastly, the crosswind profiles using the MM5 numerical weather prediction model show a large positive bias of $3.87 \mathrm{~m} / \mathrm{s}$ in the predicted crosswinds when compared to the baseline wind estimates at SFO. At San Francisco airport, a negative crosswind is a wind from the San Francisco Bay (bay wind) and a positive crosswind is a land wind. Thus the MM5 simulation data exhibits a land wind bias.

It is frequently possible to eliminate a mean bias from a data set using simple correction factors. These corrections may, or may not, improve the precision of the individual estimates. The crosswind residual data sets shown in this paper all exhibit systematic variation in the bias estimates which are a function of the baseline crosswind magnitude.

Table 2 shows how the distribution of the bias in the proxy crosswind estimation method varies as a function of the baseline crosswind magnitude. The box plots shown in the table are a convenient way of depicting groups of numerical data through a simple graphical summary: the whiskers show the sample minimum and maximum, the box shows the range between the lower quartile and upper quartile and contains $50 \%$ of the data with $25 \%$ of the residuals above and $25 \%$ of the residuals below, and the red line indicates the sample mean. The darker gray line is the median of the sample. The dataset has not been processed to remove outliers in the wake vortex observations or in crosswind estimates. 
Table 2 indicates that there is a systematic bias in the proxy crosswind that is a function of the magnitude of the baseline crosswind. Bias is calculated as the average of the proxy crosswind minus the baseline crosswind over the

Table 3. Comparison of the mean standard deviation, and its standard deviation, in the crosswind for each of the methods as compared to the baseline crosswind. The mean standard deviation provides an estimate of the amount of difference in the shape or form of the vertical crosswind profile.

\begin{tabular}{|c|c|c|c|c|c|c|c|c|}
\hline & \multicolumn{2}{|c|}{ bilinear } & \multicolumn{2}{|c|}{ proxy } & \multicolumn{2}{|c|}{ ASOS } & \multicolumn{2}{|c|}{ MM5 } \\
\hline & $\begin{array}{c}\text { Mean } \\
\text { std } \\
(\mathrm{m} / \mathrm{s}) \\
\end{array}$ & $\begin{array}{c}+/- \\
\text { std } \\
(\mathrm{m} / \mathrm{s})\end{array}$ & $\begin{array}{c}\text { Mean } \\
\text { std } \\
(\mathrm{m} / \mathrm{s})\end{array}$ & $\begin{array}{c}+/- \\
\text { std } \\
(\mathrm{m} / \mathrm{s}) \\
\end{array}$ & $\begin{array}{c}\text { Mean } \\
\text { std } \\
(\mathrm{m} / \mathrm{s}) \\
\end{array}$ & $\begin{array}{c}+/- \\
\text { std } \\
(\mathrm{m} / \mathrm{s}) \\
\end{array}$ & $\begin{array}{c}\text { Mean } \\
\text { std } \\
(\mathrm{m} / \mathrm{s})\end{array}$ & $\begin{array}{c}+/- \\
\text { std } \\
(\mathrm{m} / \mathrm{s}) \\
\end{array}$ \\
\hline DEN'06 $(n=4372)$ & 0.10 & 0.11 & 0.10 & 0.08 & 0.17 & 0.18 & & \\
\hline DEN'03 $(n=1966)$ & 0.10 & 0.09 & 0.15 & 0.10 & & & & \\
\hline $\mathrm{SFO}(\mathrm{n}=2151)$ & 0.08 & 0.07 & 0.15 & 0.11 & & & 0.33 & 0.29 \\
\hline STL (n=2237) & 0.12 & 0.11 & 0.13 & 0.09 & 0.19 & 0.15 & & \\
\hline
\end{tabular}

height interval where the wake vortex was observed; so the negative (positive) mean bias shown for positive (negative) crosswinds indicates that the proxy crosswind tends to be less than the baseline crosswind estimates. The magnitude of the proxy crosswind tends to be approximately 5\% less than the baseline crosswind over the entire range crosswinds sampled. The difference in the crosswind estimates may be caused by (1) a bias in the lidar lineof-sight wind that is a function of the magnitude of the crosswind, or (2) a bias in the wake lateral position estimates provided by the wake vortex algorithm that is a function of wind speed or (3) some physical feedback in the wake vortex physics that would decrease the wake lateral propagation speed of the vortex pair in the presence of a crosswind, but is not included in the proxy method. If the linear trend in the mean bias is removed, the standard deviation of the bias distributions also reduces by modest amounts to $0.39,0.39,0.32$, and $0.43 \mathrm{~m} / \mathrm{s}$ for the DEN'06, DEN'03, SFO and STL data sets, respectively.

Table 2 also shows that the standard deviation, or spread, of the bias distribution is not a strong function of the baseline wind speed for the proxy crosswind estimates. Thus the precision of the proxy method is approximately the same at all wind speeds, even though there is a systematic variation in the accuracy of the mean bias estimate which is a function of the wind speed.

Similar to the proxy crosswind method, it is possible to remove the mean bias in the ASOS crosswind prediction method using a linear function dependent on the magnitude of the baseline crosswind estimate. Again the trend is that the ASOS wind estimate is less than the baseline crosswind estimate, in these case by $34 \%$ and $8 \%$ for DEN'06 and STL, respectively. There is also a more substantial mean offset of -0.56 and $-0.2 \mathrm{~m} / \mathrm{s}$, respectively, for each of the datasets. If the mean bias is eliminated using these linear relationships, the standard deviation in the mean bias distribution reduces to 1.94 and $0.9 \mathrm{~m} / \mathrm{s}$ for the DEN'06 and STL data sets, respectively. Thus the standard deviations of the mean bias distribution remain quite large for the ASOS comparisons, even after these corrections. It is possible that a more sophisticated treatment of the ASOS winds which used boundary layer theory to extrapolate the wind profile to height would produce better estimates than the extrapolation of a constant value that is used in this analysis.

For the MM5 crosswind method, there is a very noticeable time of day trend in the bias which can be removed using a simple sine function fit. After the time of day trend is removed, a trend in the mean bias still exists as a function of the baseline crosswind. Similar to the ASOS and proxy crosswinds, the MM5 simulation results predict crosswinds which are less than the baseline crosswinds; in this case approximately $40 \%$ less after the mean bias and time of day corrections are applied. Removal of these trends reduces the standard deviation of the mean bias distribution from $2.48 \mathrm{~m} / \mathrm{s}$ to $1.85 \mathrm{~m} / \mathrm{s}$. This is a substantial reduction in spread, but the spread is still more than 2 times greater than any of the other crosswind estimation methods and is quite large. These results are similar to a previous study examining differences between lidar and RUC estimated crosswinds ${ }^{15}$ that found the standard deviations of the mean bias ranging from 1.71 to $2.30 \mathrm{~m} / \mathrm{s}$ over height ranges from 20 to $220 \mathrm{~m}$.

\section{B. Mean variance in crosswind profile}

The variance of the crosswind profile differences also provides some insight into the estimation error of each method and the uncertainties in the meteorological sensing. When differencing two individual crosswind profile estimates, the bias provides an estimate of the shift in the mean crosswind between the two crosswind profiles. The variance of the differences is an estimate of the change in the shape or form of the two profiles. 
The difference in the shape of the vertical crosswind between different crosswind estimation methods can therefore be estimated using the mean of the variance of the difference between one crosswind estimation technique and the baseline crosswind. Table 3 shows the mean standard deviation, or the square root of the variance, for each method and for each study area.

The bilinear interpolation crosswind estimation method, which includes the information on the time history of the vortex trajectory, has mean standard deviations which range from 0.08 to $0.12 \mathrm{~m} / \mathrm{s}$. Thus, as expected, the main affect of the bilinear interpolation method is to modify the shape of the vertical profile in comparison to the baseline method, while having only a small impact on the mean wind.

The proxy crosswind estimation method has similar, but slightly larger, mean standard deviations than the bilinear interpolation method, ranging from 0.10 to $0.15 \mathrm{~m} / \mathrm{s}$. Typically the bilinear interpolation and proxy methods produce vertical velocity profiles which are similar in form or shape.

The ASOS wind profile is taken as constant with height in this study. It therefore represents the null hypothesis of zero crosswind variability with height and provides an estimate of variability of the crosswinds over the height range covered by the vortices at the different sites. The null hypothesis of zero crosswind variability can also be checked for the other data sets using any constant wind. Performing this experiment with the DEN'03 and SFO data sets yields mean standard deviations of 0.24 and 0.23 , respectively.

The reduction in mean variance for the bilinear interpolation and proxy method compared to the ASOS or null hypothesis indicates some predictive capability for these methods in estimating the shape or form of the baseline vertical crosswind profile. On the other hand, the MM5 results show an increase in the mean standard deviation, and indicate that the MM5 numerical simulation frequently predict a different form or crosswind profile shape than the baseline or the other crosswind estimation techniques.

\section{Summary}

Spatial and temporal variability of the vertical crosswind profile is important in the prediction of wake vortex transport and circulation decay. In this paper, we compared several different techniques that are used to estimate vertical crosswind profiles to drive fast-time wake vortex prediction models. The results of this study support previous work that indicated a high level of accuracy for LMCT pulsed lidar line-of-sight wind estimates. In this study, the accuracy of the lidar line-of sight winds can be inferred by comparison of the proxy crosswind estimates, which do not directly include the lidar wind estimates, and both the baseline and bilinear interpolation crosswind estimates. The mean difference in the proxy crosswinds from the baseline and bilinear interpolation method are less than $0.30 \mathrm{~m} / \mathrm{s}$ at each site. Furthermore, the difference in the mean crosswinds for the proxy method is found to be caused by the proxy winds being approximately $5 \%$ less than the baseline crosswind over the entire range of wind speeds observed. Thus the mean bias between the proxy crosswinds and the lidar measured winds is merely a function of the wind distribution at the different sites. The ASOS and MM5 methods do not utilize any in-situ lidar observations and have substantially larger standard deviations in their bias estimates (from 0.92 to $2.5 \mathrm{~m} / \mathrm{s}$ ) than other crosswind estimation techniques (standard deviations of up to $0.51 \mathrm{~m} / \mathrm{s}$ ). Large variances in the crosswind bias indicate uncertainty on the mean wind estimate from one observation interval to the next, even if the mean bias of the entire dataset is small. We also note that temporal variability in the mean wind is approximately the same magnitude as the spatial variability in the vertical crosswind profile over the heights traversed by the vortices during the observation time interval. Thus both the mean shift of the wind and changes in the shape of the wind profile are important when estimating uncertainties in the vertical crosswind profiles relevant to wake vortex studies.

\section{Acknowledgement}

This work was sponsored by the National Aeronautics and Space Administration under NASA NRA Enabling Super-Dense Operations by Advancing the State of the Art of Fast-Time Wake Vortex Modeling. Neil O'Connor and Dr. Fred Proctor are the technical monitors. The lidar data used in this study was provided by the NASA and the Federal Aviation Administration (FAA).

\section{References}

${ }^{1}$ Holzäpfel, F., "Probabilistic Two-Phase Wake Vortex Decay and Transport Model”, Journal of Aircraft, Vol. 40, 2003, pp. 323-331.

${ }^{2}$ Proctor, F.H., Hamilton, D.W., and Switzer, G.F. “TASS Driven Algorithms for Wake Prediction,” AIAA Paper 2006-1073.

${ }^{3}$ Proctor, F.H., “Evaluation of Fast-Time Wake Vortex Prediction Models,” January 2009, AIAA Paper 2009-0344. 
${ }^{4}$ NextGen Integration and Implementation Office, FAA's NextGen Implementation Plan March 2010, URL: http://www.faa.gov/nextgen/media/NGIP_3-2010.pdf [cited 27 November 2010].

${ }^{5}$ Robasky, F. M., Clark, D. A., A "Wind Forecast Algorithm to Support Wake Turbulence Mitigation for Departures (WTMD)", 13th Conference on Aviation, Range, and Aerospace Meteorology (ARAM), New Orleans, LA, Amer. Meteor. Soc., 2008.

${ }^{6}$ Holzäpfel, F., "Minutes of the WakeNet3-Europe Workshop on Short-Term Weather Forecasting for Probabilistic WakeVortex Prediction", Institut für Physik der Atmosphäre, Duetsches Zentrum für Luft- und Raumfahrt. April 2010.

${ }^{7}$ McGinley, J. A., S. C. Albers, P. A. Stamus, "Local Data Assimilation and Analysis for Nowcasting”, Advances in Space Research, Vol. 12, 1992, pp. 179-188.

${ }^{8}$ Klemp, J. B., W. C. Skamarock, and J. Dudhia, “Conservative Split-Explicit Time Integration Methods for the Compressible Nonhydrostatic Equations", WRF Eulerian Prototype Model Equations Draft Manuscript, National Center for Atmospheric Research, 2000.

${ }^{9}$ Bacon, D. P., N. N. Ahmad, Z. Boybeyi, T. J. Dunn, M. S. Hall, P. C. S. Lee, R. A. Sarma, M. D. Turner, K. Waight, S. Young, and J. Zack, “A Dynamically Adapting Weather and Dispersion Model: The Operational Multiscale Environment Model with Grid Adaptivity (OMEGA)”, Monthly Weather Review, Vol. 128, 2000, pp. 2044-2076.

${ }^{10}$ Hodur, R. M., “The Naval Research Laboratory's Coupled Ocean/Atmosphere Mesoscale Prediction System (COAMPS)”, Monthly Weather Review, Vol. 125, 1997, pp. 1414-1430.

${ }^{11}$ Benjamin, S. G., G. G. Grell, J. M. Brown, T. G. Smirnova, R. Bleck, "Mesoscale Weather Prediction with the RUC Hybrid Isentropic-Terrain-Following Coordinate Model”, Monthly Weather Review, Vol. 132, 2004, pp. 473-494.

${ }^{12}$ Kaplan, M. L., Y. Lin, C. Ringley, Z. Brown, M. Kiefer, P. Suffern, A. Hoggarth, "Wake Vortex Environment Simulations from a Terminal Area PBL Prediction System (TAPPS)”, AIAA Paper 2006-1074.

${ }^{13}$ Frech, M., F. Holzäpfel, A. Tafferner, T. Gerz, "High-Resolution Weather Database for the Terminal Area of Frankfurt Airport”, Journal of Applied Meteorology and Climatology, Vol. 46, 2007, pp. 1913-1932.

${ }^{14}$ Frech, M., F. Holzäpfel, "Skill of an Aircraft Wake-Vortex Model Using Weather Prediction and Observation”, Journal of Aircraft, Vol. 45, 2008, pp. 461-470.

${ }^{15}$ Huang, S., Robasky, F. M., Clark, D. A., "Comparison of Rapid Update Cycle (RUC) Model Crosswinds with LIDAR Crosswind Measurements at St. Louis Lambert International Airport", 13th Conference on Aviation, Range, and Aerospace Meteorology (ARAM), New Orleans, LA, Amer. Meteor. Soc., 2008.

${ }^{16}$ Hastie, T. J. and Tibshirani, R. J., "Generalized Additive Models”, London, Chapman \& Hall, 1990.

${ }^{17}$ Dudhia, J., "A nonhydrostatic version of the Penn State/NCAR mesoscale model: Validation tests and simulation of an Atlantic cyclone and cold front", Monthly Weather Review, Vol. 121, 1993, pp. 1493-1513. 